\title{
Lapurdum
}

LAPURDUM Euskal ikerketen aldizkaria | Revue d'études basques |

Revista de estudios vascos | Basque studies review

$4 \mid 1999$

Numéro IV

\section{Harrieten hiztegia eta euskalkiak}

\section{Arantxa Hirigoyen}

URL : http://journals.openedition.org/lapurdum/1548

DOI : 10.4000/lapurdum.1548

ISSN : 1965-0655

Éditeur

IKER

\section{Édition imprimée}

Date de publication : 1 octobre 1999

Pagination : 115-121

ISBN : 2-84127-156-0

ISSN : 1273-3830

\section{Référence électronique}

Arantxa Hirigoyen, « Harrieten hiztegia eta euskalkiak », Lapurdum [Linean], 4 | 1999, Sarean emana--an 01 avril 2010, kontsultatu 01 février 2020. URL : http://journals.openedition.org/lapurdum/1548 ; DOI : 10.4000/lapurdum.1548 


\section{Arantxa HIRIGOYEN}

\section{HARRIETEN HIZTEGIA ETA EUSKALKIAK}

Tesi lan gisa prestatzen ari naizen ikerlanak, Maurice Harriet (1814-1904) haltsuarrak bere eskuz landu zuen euskara-frantsesa hiztegi argitaratugabea du aztergai. Harriet deitura aipatzen delarik, Martin Harriet gramatikalariari, bere aitatxi zenari hain xuxen, pentsatzen du jende frankok. Hemen aldiz, mende hastapen huntan zendu den hiztegigile lapurtar batez mintzo naiz. Ene iduriko, Maurice Harriet haltsuar apezak ez du oroitzapen handirik utzi gure artean, isilpean joan zaigu. Alta, 3536 hosto dituen hiztegi oso bat utzi digu ondare gisa. Bere bizitzako azken 30 urteak euskara-frantsesa hiztegiaren eraikitzen iragan omen zituen Harrietek. Ikaragarriko lan honek ez ote luke oihartzun gehiago merezi ? Egia da, hiztegi baten baliogarritasuna eta ekarpena neurtzea zaila dela, bainan halaere hiztegi hortaz baditeke zer erran eta zer iker. Zendako ez ote da sekulan argitaratu ? Maurice Harrietek bere iturri garbiak ukan ditu, bainan azpimarra dezagun ere, haltsuar hiztegigilea bera ere, iturri gisa erabilia izan dela. Mende huntako zenbait hiztegigileen hiztegietako iturrien zerrendan agertzen baita haltsuarraren lana : R.M. Azkue, P. Lhande, K. Mitxelenaren lanetan adibidez.

Artikulu hunen xedea bikoitza da. Batetik hiztegiaren azala edo itxura ikertuko dugu, ezaugarri nagusiak aipatuz. Bigarrenik, aldiz, hiztegiaren mamian sartuko gara, eta atal berezi batetaz solas eginen dugu. Euskara-frantsesa hiztegia izanik, ikusiko dugu zer-nolako garrantzia eta lekua duten euskalkiek hiztegian.

Iduritzen zait azala eta mamia bi hitz hauek laburbiltzen dutela haltsuarraren hiztegia. Azala, erabil nezake hiztegi horrek duen itxurarengatik. 3.536 orriko hiztegi batek, kanpotik bederen, bada zer aipa. Ez da edozein hiztegi arrunt, itxuraz hasteko. Hiztegiaz bertzalde ohartarazi nahi nuke oharren liburuxka bat ere aurkitzen dela, Cahier de notes izenburupean. 50 orri baino gehiagoz osatua den kaier bat da. Delako oharren liburuxka horretan orden alfabetiko batean sailkatuak dira orriak A letratik Z letraraino. Hitzak zerrendatuak dira bat bertzearen gibeletik eta sarrera hitzaren ondotik gehienetan sinonimo bat ematen du Harrietek edo frantsesezko itzulpena agertzen da. Liburuxka horretan ez du xehetasun handirik ematen. Gainera behin baino gehiagotan berriz hasten du orden alfabetikoa bertze hitz zerrenda batekin. Bi gogoeta heldu zaizkit burura idazki berri horri so eginez : edo eranskin gisa nahi izan zuen erabili kaier hau haltsuarrak, edo hiztegia egiten ari zuen heinean, ondoan, delako liburuxka horretan, ahanzten zituen hitzak markatzen zituen.

Bertzalde, Harrietek berak eskuz idatzi bibliografia baten zerrenda bada, bai eta idazmakinaz izkiariatu kopia bat ere ; azken hau, hain segur ere, Lhanden hiztegia prestatzen ari zirenen garaikoa dateke. 
hamia, aldiz, edukiaren aldetik erran nezake. Maurice Harrieten hiztegiak edozein hiztegik eskaintzen duen informazio mota biltzen du. Ondoko ezaugarriek beztitzen dituzte sarrera-hitzak : mugabideak, adibideak, sinonimoak, etimologiak, hitzaren hedadura eta beste irizpide franko ageri da artikuluetan.

Euskaratik frantseserako hiztegia izanik, hiztegiko hitzen sarrerak euskaraz agertzen dira, eta mugabideak frantsesez. Hots, frantsesez esplikatzen ditu euskal hitzak. Hala ere batzuetan euskal hitzaren baliokidea bertze zenbait hizkuntzetan ematen du, hitzaren jatorriaz mintzo delarik bereziki : gaztelera, gaskoina, portugesa edo italiera adibidez. Adibideak aiseago hatzeman ditugu T letran A letran baino. T letran, erdal jatorria duten hitz gehiago aurkitzen baita. Hala nola $\boldsymbol{T A C A}$ sarrera-hitzan, mugabidearen eta adibidearen ondotik hitz horren itzulpena beste hizkuntzetan irakurtzen ahal da

"Le patois roman et les langues romane's, emploient souvent au sens de tache, souillure, [..], esp.taca- it tacca-port.tacha-fr.tache-picard take [...]".

TAPITCEA hitzaren etimologia aipatzen duelarik ere ondoko ohar hau eransten du : "[...] il faut en effet en attribuer son origine commune avec tapes, tapotis lat. tapis couverture-tapis fr. tapiz esp. port-tapietto it-tapi. prov. tapis [...]"

THUÑ $A$ : [...] Le gascon dit [...] taugna-taugnade, donner un coup, coup porté à poing fermé : y aurait-il un emprunt du basque au gascon, ou du gascon au basque? [...].

Gisa honetako adibide franko irakurtzen ahal da hiztegian, etimologiaz ari denean bereziki. Erdal jatorria duten hitzak agertzean, hitzaren etorkia ematen du Harrietek, eta orduan dira ageri bertze hizkuntzak. Gaurkoan, ez dugunez etimologia aipagai, ez gara hortan luzatuko.

Hiztegi honek duen ezaugarri nagusi bat, ene ustez, euskalkien aipamena da. Hiztegigileak euskalki guziak konduan hartzen ditu edo aipatzen ditu bederen. Horrek erakusten du Harrietek mugaz bertzaldeko euskalkiak ezagutzen eta menperatzen zituela. Oroitaraz dezagun Madrilen egon zen urte haietan (1855-1878) Maurice Harrietek, Jose Francisco Aizkibel hiztegigile azkoitiarra ezagutu zuela eta adixkide handi bilakatu zirela. Gaztelera biziki ongi menperatzeaz gain hego Euskal Herri osoko euskalkiak hobeki ezagutzeko eta lantzeko parada izan zuen, han ezagutu zituen euskal unibertsitariei esker.

Erran bezala, sarrera-hitzaren ondotik kasik beti, aldakiak agertzen dira, erran nahi baitu beste euskalkietan erabiltzen diren formak idazten dituela Harrietek sinonimo gisa. Aldaki horien ondoan maiz zein euskalkitik etortzen diren azpimarratzen du laburduren bidez: $\mathrm{s}$. = zuberera, 1 . = lapurtera, BN. = behenafartarra, b. = bizkaiera, g. = gipuzkera, Nav. = nafarrera, $\mathrm{HN}$. = goi-nafarrera. Harrietek erabiltzen dituen laburdura hauek euskalkien sailkapena islatzen dute. Huna hemen A eta T letretan bildu ditudan zenbait adibide erakusgai gisa :

Achiruina: l. id. achiroina, achoina Sy. zango cabila l., zango-aztala-aztalbeharria : BN, S. cheville du pied : id. g.b. isuriña-churminoa, orgatilla, oindagora, izuriña. [...]

Aguia : [...] 3. Aguian [..] Sy. l.g. behar bada, menturaz, BN, S. heldu, heltu bada, b. ausaz, ausa, aiyaz, apercon bai, b. beharbada bai l.g.BN.S. - nasqui, nabasqui g. noasqui g.b. [...]. 
Ainhara : l., ainhera $B N$, S., inhara l., enada, elaia, ainada g.b. [...]

Akhetza, akhetcha I. BN. verrat-Sy. BN. ordotsa BN.S., cherri akhetza, berreta S., cherri-ordotsa, ordotcha, arkotcha g. Izt.G.C., apotea g. [...]

Ardoa g. ardaua b. arnoa l.BN, anoa, anhoa, S.BN-le primitif est ardo, comme l'emploient le g. et b., et ainsi que l'indiquent ses composés dans les autres dialectes: ardanasca, ardantza, etc-.

TACADA : BN. tranchée : Sy. erretena, g. erreca L.BN.S.[...]

I TANDA : [...] Sy. chorta l.BN.s., ikhina lab.- tanga b.- tangoa g.Nav-[...]

TAPATCEA, tapa, tapatu, tapatcen [...] Sy. herstea, l. erstea, g. ichtea b. [...]

TELA : [...] Sy. euta g.b. (de eotcea, eo, tisser). Oihala, oiala, l.BN.s.[...]

TRUFA : [..] Sy. burla, moquerie par paroles ou gestes-escarnioz, même sens, et geste moqueur-iseca g.b. iñaquiña g.b. ihaquina l.BN.S. : geste de moquerie- $[\ldots]$

Maiz euskalkiei buruzko oharrak hatzematen ahal dira hiztegian. Euskalkien arteko berezitasunak eta ezaugarriak aipatzen ditu, hitzaren erabilpena, hedadura eta ahozkerari buruzko xehetsunak emanez. Lapurdiko, Zuberoako eta Nafarroa Behereko euskalkien arteko bereizkuntza nahiz, orohar, euskalkien arteko ezaugarriak ezagutarazten ditu haltsuarrak. Bi multzotan sailkatu ditugu adibideak. Lehen sailan, lapurtera, zuberera eta behe-nafarrerari buruzko zenbait ohar kokatu ditugu:

TAINQUI : [...] Le labourdin met une différence entre tinqui, avec force, et tainqui beaucoup.

TANTAIA : //I. Proprement branche d'arbre [...]//4. En BN il signifie, pieu, paisseau, servant à soutenir un pied de vigne: Sy. l. phaldoa, phacheta [...].

TARRA, suff. DARRA après $n$, arra après une voyelle : qui est originaire, ou habitant de : [...]. Le souletin change parfois tiarra en liarra : ezteiliarra, pour eateitiarra: convive en une noce.

TCEA : Suff. //l. Suffixé à un nom, il signifie : grand nombre, un amas : [...] //2. En souletin et BNav. terminant un nom d'arbre, il signifie, pied, plant de:

Ficotce bat, un figuier [...].

TCEN ou TEN : Suff. verbal. [...] // dans les verbes qui régulièrement devraient être zten, le Bas-navarrais surtout dit tcen par métathèse : hazten, hatcen- hozten, hozten (sic)- lazten, latcen [...].

THINICATCEA, thinica, thinicatu, thinicatcen : écimer. [...]-Thinia et ses dérivés, particuliers à la Soule et une partie de la BN paraissent termes purement basques, se trouvent dans ces seuls dial. et dans aucun autre, et ne se rencontrent pas dans les langues romanes, ou dans le latin.

TIPULA, id. quipula : Oignon, cibule- Sy. le Soul. dit encore cepola, et uñhua. [...]

TITATCEA, tita, titatu, titatcen : même sens que titacatcea, qui est aussi le même mot, avec particule dimin. ca-Sy. Le Labourdin a pincardatcea-picadartcea, le $B N$ et le Souletin ont picardatcea, picaillartcea. [...] 
TREMPEA : Silv. Pouvr. id. trempua v., plus usité en labourd (sic), trempea en BN.S.

TRESNATCEA, tresna, tresnatu, tresnatcen : [...] Ce verbe appartient aux dial. cispyr., spécialement au Bas-Navarrais et au Souletin, où il est d'usage habituel.

Erran bezala, Maurice Harrietek mugaz bi aldetako berezitasunak aipatzen ditu hiztegi honetan. Oharren ala iritzien bidez, hiztegigileak, dialectes cispyrénéens eta dialectes transpyrénéens izendapenekin bereizten ditu, ipar eta hego Euskal Herriko euskalkiak. Eskaintzen dituen xehetasunen bidez, euskalkien arteko harremanak eta ezberdintasunak agerian uzten ditu, ondoko etsenplu zerrendan ikusiko dugun bezala :

Akhetza, akhetcha : I. BN. verrat- [...] Akhetza, proprement, exprime mâle en général ; comme ordotsa, orotsa ; cela ressort de ce que les dialectes g. et BN. le font synonyme de ordotsa, lequel signifie quadrupède mâle [...].

Arguizaguia, arguizaria : [...] Le Biscayen et le BN. disent arguizaia par contraction - [...] on ne trouve nulle part que zaguia avec une signification propre ou employé seul : le Biscayen a bien Zaguia, mais c'est une contraction de zahaguia, outre- Darrigol, dans sa dissertation fait dériver la terminaison Zaria de izaria, mesure : mais autre que cette terminaison n'est propre qu'au dialecte Bas-Navarrais, qui même n'en use pas toujours, argui-izaria mesure de lumière, pour dire lune, parait (sic) plutôt une étymologie ingénieuse et forcée, qu'exact:' [...]: néanmoins elle n'est pas tout à fait à rejeter [...].

I.TARA : l. Suffi. // . Signifie plein ou contenant : besotara, ahurtara [...]// Souvent, sans accompagnement de substantif, il signifie en général, une forte quantité ; le nom de la chose étant sous-entendu: [...] Sy. eta, ta : BN.S. zareta, ahurreta, panérée- Le g. et le b. emploient le substantif sans suffixe suivi du nom de la chose contenue; comme d'ailleurs il est usuel dans tous les dialectes: sasqui bat aran : une corbeille, une corbeillée de prunes.

TCHO : suff. diminutif : [..]. Habituellement les dial. l.BN.S. écrivent ce diminutif par deux $\boldsymbol{t}$, tto et le prononcent comme un $\boldsymbol{t}$ mouillé; les dial. gret $b$. disent et écrivent tcho, tchu, par une prononciation fort approchante du tto l. [...].

II.TRESNA [...] I. habits, vêtements, effets, hardes. [..]. Ety. tresna au sens de vêtement est un néologisme appartenant aux dial. cispyrénéens auxquels les dial. transpyr. l'ont emprunté (85.or) et dont ils font un rare usage, excepté dans la Haute Navarre, et pays limitrophes des pays basques fr. [...].

II.TRUCA : [...] troc, échange : Il s'emploie à l'indéfini dans les dial. cispyrénéens. [...] Les dial. transpyrénéens l'emploient de même fréquemment à l'indéfini, mais aussi ils le déclinent. [...].

TRUMPATCEA, trumpa, trumpatu, trumpatcen : [...] Ce verbe néologique, appartenant aux dial. cispyrénéens y est fort usité, ainsi que des dérivés : il a la plupart des acceptions du verbe français: on fait usage habituel dans tous les dialectes d'un autre verbe, aussi néologique : enganatcea, esp. engañar. [...]. 
Euskalkiaz gain, batzuetan, herrien izen zehatzak ematen ditu Harrietek. Norbaiti entzunak ala berak bilduak ote dira? Huna hemen zenbait adibide, A letran bilduak :

Ahia $l$. BN. S., b. g. aia : bouillie [...] Sy. cihaña (Ste Engrace) (sic) [...]

Aintcia b. / Aintea, g.b. id. aintea b.g. force, vigueur, (syncope de aguintea, v.) - Aintcitsua, antcitsua, fort, vigoureux [...]-// cer aintci da? Quelle heure estil? ou cer aintce? (Bardos-Arraute) [...]

Akhuiliac (beguien-) lunettes (Ste Engrace) (sic)

Aratchea $l$. [...] Aratche chahal-çahal-caicu-burua: grenouille à l'état de têtard : id. Chahal bohoca S., igueltchoa g.b. - butchapula, Hasparr.

Adibide guzi hauetan barna, Maurice Harrietek hiztegia egiterakoan hautatu duen irizpide bat ageri zaigu. Hots, hiztegigileak euskalki nagusiak konduan hartu ditu bere lana prestatzeko tenorean. Dudarik ez da hortarako, eskura zituen liburu garrantzitsu guziak edo gehienak erabili eta miatu zituela. Lekukotasunak aho eta luma hizkuntzakoak dira. Bertzalde, Harrietek berak ere zenbait hiztegi baliatu ditu ; Larramendi, Pouvreau eta Sallaberry hiztegigileen obren aztarnak ikusten ahal dira hiztegi osoan barna. Azkenik, Madrilen iragandako egonaldia eta han egin dituen ezagutzak biziki lagungarri izan zaizkiola ezin uka.

Erakusgai ttipi hunen bitartez, Maurice Harrieten euskara-frantsesa hiztegian barna murgildu gara. Ene iduriko, hiztegi horrek gordetzen duen aberastasun bat ezagutu dugu. Hiztegi elebiduna bada ere, euskal hizkuntzari begira, orohar, ekarpen ugari egiten ditu, euskalkien lexikoari leku handi bat utziz. Hots, euskalkien sailkapena eta euskal hizkuntzaren aldakiak berezi ditu eremu zabal batean.

\section{Résumé}

Cet article constitue une contribution à la façon dont M. Harriet (1814-1904) traite de la question des dialectes dans son dictionnaire manuscrit et resté inédit, gros de 3536 pages et dont l'auteur a fait son sujet de thèse (en cours). Après avoir fourni des indications sur la micro-structure de ce dictionnaire, ce travail montre comment Harriet distingue "dialectes cispyrénéens" et "dialectes transpyrénéens" mais recourt parfois à une localisation plus précise jusqu'au niveau du village quand une forme y est endémique. La thèse devrait permettre de mieux repérer les sources utilisées par Harriet en plus de Larramendi, Pouvreau et Sallaberry dont l'influence est manifeste.

Arantxa HIRIGOYEN (Doctorante en Études basques) 


\section{Bibliografia}

- Azkue, R.M. de, Diccionario Vasco Español Frances, Euskaltzaindia, Bilbo, 1984.

- Arzamendi, J., 1981, "Euskal Lexikografiaren historiarako hastapenak", Euskal linguistika eta literatura : bide berriak. Deustuko Unibertsitatea, Bilbo, 163-193.

- Azkarate, M., 1994, "Oihenart-en Lexiko-sorkuntza" Iker-8, 49-77. Bilbo : Euskaltzaindia.

- Bilbao, J., 1970, Eusko Bibliografia, Donostia-Bilbo : Auñamendi (13 lib),

- Bilbao, G., 1992, "Pouvreauren hiztegi laukoitza" ASJU XXVI-2, 341 -389.

- Chaho, A., 1856, Dictionnaire Basque Français, Espagnol et Latin, Baiona.

- Daranatz, J.B., 1908, "Le Testament Berria, de Haraneder, et ses éditeurs les abbés Dassance et Harriet", RIEV, 150-177. $457-462$.

Daranatz, 1923, "Le dictionnaire Basque Espagnol Français de l'abbé R.M. de Azkue", RIEV XIV,

- Daranatz, J.B., 1929, "Correspondance du Capitaine Duvoisin", RIEV 20, 152-181.

- Etxebarria, J.M., 1990, "XVIII eta XIX. gizaldietako sei hiztegi argitaragaberen berri", in E.Perez \& P. Urkizu (arg.), Patxi Altunari Omenaldia, 63-83, Deustuko Unibertsitatea-EUTG, Donostia.

- Imbs, P., 1971, "Le Tresor de la Langue Française". Bulletin de la Société de Linguistique, 85-106.

- Kerejeta, M.J,, 1991, "Oihenart Silvain Pouvreauren hiztegian”, ASJU XXV-3, 865-889.

- Knörr E., 1985, "Maurice Harriet-en hiztegiaz", ASJU, 41 3-417.

- Knörr E. 1987, Para una edición critica del diccionario de Maurice Harrier. EHU (Euskal Herriko unibertsitatea) / Université du Pays Basque, Vitoria/Gasteiz (tesi argitaragabea).

- Lafitte, P., 1965, "Cinq dictionnaires basques". In Bulletin du Musée Basque 30, 17 1-176.

- Lakarra, J.A., 1987. (argitaratzailea), Hiztegiak ela Testuak, Bilbo

- Lakarra, J.A., 1991, "Testukritika eta hiztegiak : Harriet eta Larramendi". ASJU, Gehigarrak, XIV., $217-258$.

- Lakarra, J.A., 1992a, "Larramendirekin aurreko hiztegigintzaren historiaz", Manuel Larramendi. Hirugarren mendeurrena. 1690-1990. Andoian, 279-312.

- Lakarra, J.A., 1994, "Harrieten Gramatikazko hiztegiak", ASJU XXVIII-1, 1-178.

- Lakarra, J.A., 1995, "Pouvreauren hiztegiez eta hiztegigintzaren historiaz", ASJU, 1-52

- Lakarra, J.A., 1996, "Lexiko berrikuntza euskal hiztegigintza zaharrean : zenbait ikergai", UZTARO (19. zenbakia) 3-40.

- Larramendi, Manuel de., 1745, Diccionario Trilingüe del Castellano, Bascuence y Latin. San Sebastián, B. Riesgo y Montero.

- Lhande, P., 1926, Dictionnaire Basque-Français, Parise, Gabriel Beauchesne.

- Michelena, L., 1970, Estudio sobre las fuentes del diccionario de Azkue. Bilbo.

- Michelena L., 1987, Diccionario General Vasco. Orotariko Euskal Hiztegia, Bilbo, Editorial Desclée de Brouwer, ediciones Mensajero.

- Mùgica, Plácido, 1964, Diccionario vasco-castellano, San Sebastián, Auñamendi.

- Orpustan, J.B., 1993, Oihenarteǹ hiztegia. Baigorri. Editions Izpegi.

- Orpustan, J.B., 1996, Précis d'histoire littéraire basque. Baigorri. Editions Izpegi.

- Pikabea J., 1993, Lapurtera idatzia (XVII-XIX). Bilakaera Baten Urratsak, Donostia.

- Quemada, B., 1967, Les dictionnaires du français moderne 1539-1863, Paris, Didier. 
- Rey-Debove, J., 1970, La lexicographie. Langages, Didier/Larousse.

- Rey-Debove, J., 1971, Etude linguistique et sémiotique des dictionnaires français contemporains, Paris, Mouton.

- Rey, A., 1980, La lexicologie. Paris, Klincksieck.

- Rey A., 1982, Encyclopédies et dictionnaires. Que sais-je ? n² 2000 PUF.

- Sallaberry, 1857, Vocabulaire de mots bas-navarrais traduits en langue française. Bayonne, Lamaignère.

- Sarasola, I., 1996, Euskal hiztegia, Kutxa Fundazioa, Donostia.

- Urkizu Sarasua P., 1971, "Tresora hirur lenguayetakua. Frantzesa, Españoli eta Heskuara"., ASJU V, 175-191.

- Urkizu Sarasua P., 1988, "Pierre d'Urteren Hiztegia". Hiztegiak ela Testuak, Joseba Lakarra Andrinua, (ed.), Bilbo, Servicio Editorial U.P.V., 75-99

- Urkizu Sarasua, P., 1989, Pierre d'Urteren hiztegia- Londres 1715, Donostia, Mundaiz.

- Villasante Fr. Luis, 1961, Historia de la literatura vasca, Bilbao, Sendo.

- Villasante Fr. Luis, 1973, Axular-en Hiztegia, Oñati, Jakin.

-Vinson, J., 1910, "Le vocabulaire de Pouvreau", RIEV 4, 139-141. 